3 Falzon D, Jaramillo E, Schünemann HJ, et al. WHO guidelines for the programmatic management of drug-resistant tuberculosis: 2011 update. Eur Respir J 2011; 38: 516-528.

4 Gumbo T, Louie A, Deziel MR, et al. Selection of a moxifloxacin dose that suppresses drug resistance in Mycobacterium tuberculosis, by use of an in vitro pharmacodynamic infection model and mathematical modeling. J Infect Dis 2004; 190: 1642-1651.

5 Liang H, Kays MB, Sowinski KM. Separation of levofloxacin, gatifloxacin, moxifloxacin, trovafloxacin and cinoxacin by HPLC: application to levofloxacin determination in human plasma. J Chromatogr B Analyt Technol Life Sci 2002; 772: 53-63.

6 Nightingale $\mathrm{CH}$. Moxifloxacin, a new antibiotic designed to treat community-acquired respiratory tract infections: a review of microbiologic and pharmacokinetic-pharmacodynamic characteristics. Pharmacotherapy 2000; 20: 245-256.
7 Peloquin CA, Hadad DJ, Molino LP, et al. Population pharmacokinetics of levofloxacin, gatifloxacin, and moxifloxacin in adults with pulmonary tuberculosis. Antimicrob Agents Chemother 2008; 52: 852-857.

8 Nijland HM, Ruslami R, Suroto AJ, et al. Rifampicin reduces plasma concentrations of moxifloxacin in patients with tuberculosis. Clin Infect Dis 2007; 45: 1001-1007.

9 Pranger AD, van Altena R, Aarnoutse RE, et al. Evaluation of moxifloxacin for the treatment of tuberculosis: 3 years of experience. Eur Respir J 2011; 38: 888-894.

10 Poissy J, Aubry A, Fernandez C, et al. Should moxifloxacin be used for the treatment of extensively drug-resistant tuberculosis? An answer from a murine model. Antimicrob Agents Chemother 2010; 54: 4765-4771.

\title{
An uncommon reason for dyspnoea: phrenic paresis secondary to alveolar echinococcosis
}

\section{To the Editors:}

We describe the presentation of an Echinococcus multilocularis (EM) infection (alveolar echinococcosis (AE)) with progressive massive orthopnoea and progressive dyspnoea on exertion due to infiltration of the diaphragm and phrenic nerves. Diagnosis was proved using a combination of computed tomography (CT), lung function in the supine and sitting position, sniff test during radioscopy and $\mathrm{AE}$ serology. Because of diffuse infiltration of the retroperitoneal and mediastinal space a surgical intervention was inapplicable. Thus, medical treatment was initiated using albendazole. Noninvasive ventilation (NIV) resolved orthopnoea and the patient was discharged.

$\mathrm{AE}$ occurs in the northern hemisphere but in Switzerland it is classified as a rare disease. To our knowledge this is the first described case of this rare infection with a consecutive bilateral paresis of the diaphragm. But despite its unique entity, this case report demonstrates that clinical findings and routine diagnostic methods may lead to the correct diagnosis.

Progressive phrenic paresis is a rare cause of orthopnoea and dyspnoea on exertion [1]. Relevant paresis is often overseen and diagnosed late in the course of the disease. The main reasons for bilateral phrenic paresis are traumatic, inflammatory, neuropathic, idiopathic or tumour related [1]. We present a case of a non-malignant compression caused by AE leading to bilateral phrenic paresis.

A 60-yr-old farmer's wife presented to a district hospital with progressive dyspnoea on exertion for 2 yrs. A cardiologic consultation at that time including echocardiography and exercise testing was unremarkable. Within the last year, the patient complained about weight loss of $10 \mathrm{~kg}$ and postprandial epigastric pain. During the final 2 months before presentation, the patient developed orthopnoea, panicking in the supine position. Clinical examination revealed extenuated breathing sounds on the left basal side, but no signs for left or right heart failure. Respiration showed a paradoxical breathing pattern in the supine position. Laboratory testing was unremarkable and showed normal brain natriuretic peptide and D-dimer-values. The chest radiograph depicted an elevation of the left side of the diaphragm but otherwise was unremarkable. Further investigations (echocardiography and gastroscopy) revealed no relevant pathology. Finally, a CT scan showed a multifocal, cystoid and hypodense mass in the retrocardial, transdiaphragmal and retrocaval space (fig. 1). Because of the rapid progression of symptoms the patient was brought to our tertiary care hospital (Cantonal Hospital St Gallen, St Gallen, Switzerland) for further diagnostics and therapy.

The reason for dyspnoea and orthopnoea was considered to be either phrenic paresis or inferior vena cava syndrome due to a cystic process of unknown origin, most likely malignant or by AE.

Based on the cystoid growth a screening test for Echinococcus species (Cellognost; Dade Behring Marburg GmbH, Marburg, Germany) was performed, which showed a highly positive result. AE was confirmed by the more specific antigen test for EM (Em2plus-ELISA; Bordier Affinity Products, Crissier, Switzerland).

Inferior vena cava syndrome was ruled out using angiography and manometry. By contrast, vital capacity dropped from $47 \%$ (sitting) to $26 \%$ in the supine position (fig. 2) and orthopnoea resolved when using noninvasive positive pressure ventilation despite the normocapnic blood gases. Finally radioscopy (sniff test) confirmed functional bilateral paresis of the diaphragm.

We concluded that the dyspnoea of the patient was due to a bilateral paresis of the diaphragm caused by the infiltration of the echinococcal mass.

Because of mediastinal and retroperitoneal infiltration the infection was not considered to be surgically curable and treatment using albendazole (400 mg b.i.d.) was initiated. Treatment will continue for many years or even for life. After initiation of nocturnal noninvasive home ventilation, 

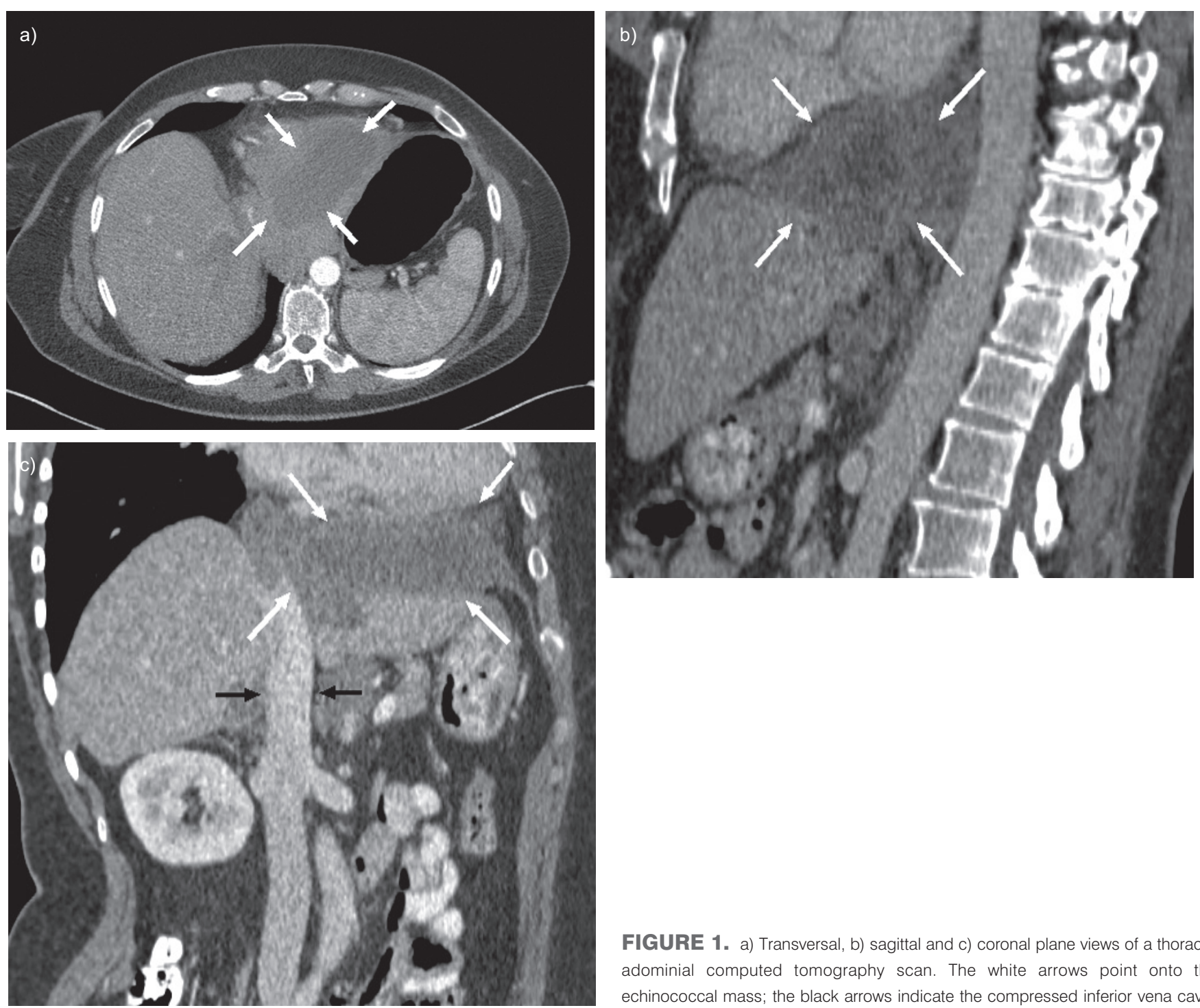

FIGURE 1. a) Transversal, b) sagittal and c) coronal plane views of a thoracoadominial computed tomography scan. The white arrows point onto the echinococcal mass; the black arrows indicate the compressed inferior vena cava.

orthopnoea resolved and the patient was discharged. She is currently followed on an outpatient basis.

To our knowledge, this is the first case report of an echinococcalrelated bilateral diaphragm paresis. AE occurs predominantly in specific regions of the northern hemisphere [2]. The primary host is the fox and intermediate hosts are small mammals. Humans are false intermediate hosts and are infected by ingestion of contaminated fruits or vegetables. Due to a successful vaccination programme against rabies the fox population in Switzerland increased four-fold between 1980 and 1995. This and the urbanisation of the foxes led to a doubling in the incidence of human AE (0.26 cases per 100,000 population) since the year 2000 [3].

Diagnosis can be made by typical findings on a CT scan but often the imaging is mistaken for a tumour due to the infiltrating character of the lesions. Serologic testing consists of a screening test for Echinococcus species with high sensitivity followed by a more species-specific test against antigens of EM (e.g. Em2plus-ELISA) [4].
Diagnosis is established if there are at least two of the following parameters fulfilled [5]: 1) typical lesions by imaging techniques; 2 ) a positive screening test with high sensitivity confirmed by a separate test with high specificity; 3) histopathology compatible with $\mathrm{AE}$; and 4) detection of $\mathrm{EM}$ nucleic acid sequence(s) in a clinical specimen.

Treatment consists of radical surgical resection accompanied by a medical therapy with benzimidazole for a minimum of 2 years. A palliative surgery with tumour debulking did not show any advantages [6]. In inoperable cases lifelong chemotherapy is recommended and the patient will be clinically monitored, including imaging and serologic monitoring, for progression. Unfortunately, this treatment is not curative because imidazoles are only parasitostatic, but survival rates are $90-94 \%$ after 10 yrs compared with $90 \%$ mortality without treatment [7].

The diagnostic approach to subacute dyspnoea generally includes early lung function testing. Even in the elderly with normal chest radiographs, cardiac function and exercise testing 


\begin{tabular}{lcccc}
\hline & \multicolumn{2}{c}{ Sitting } & \multicolumn{2}{c}{ Supine } \\
\hline FEV 1 & $1.06 \mathrm{~L}$ & $46 \%$ & $0.59 \mathrm{~L}$ & $26 \%$ \\
FVC & $1.42 \mathrm{~L}$ & $47 \%$ & $0.85 \mathrm{~L}$ & $28 \%$ \\
PI & $3.0 \mathrm{kPa}$ & $28 \%$ & & \\
PE & $5.6 \mathrm{kPa}$ & $81 \%$ & & \\
SNIP & $1.2 \mathrm{kPa}$ & $14 \%$ & & \\
\hline
\end{tabular}

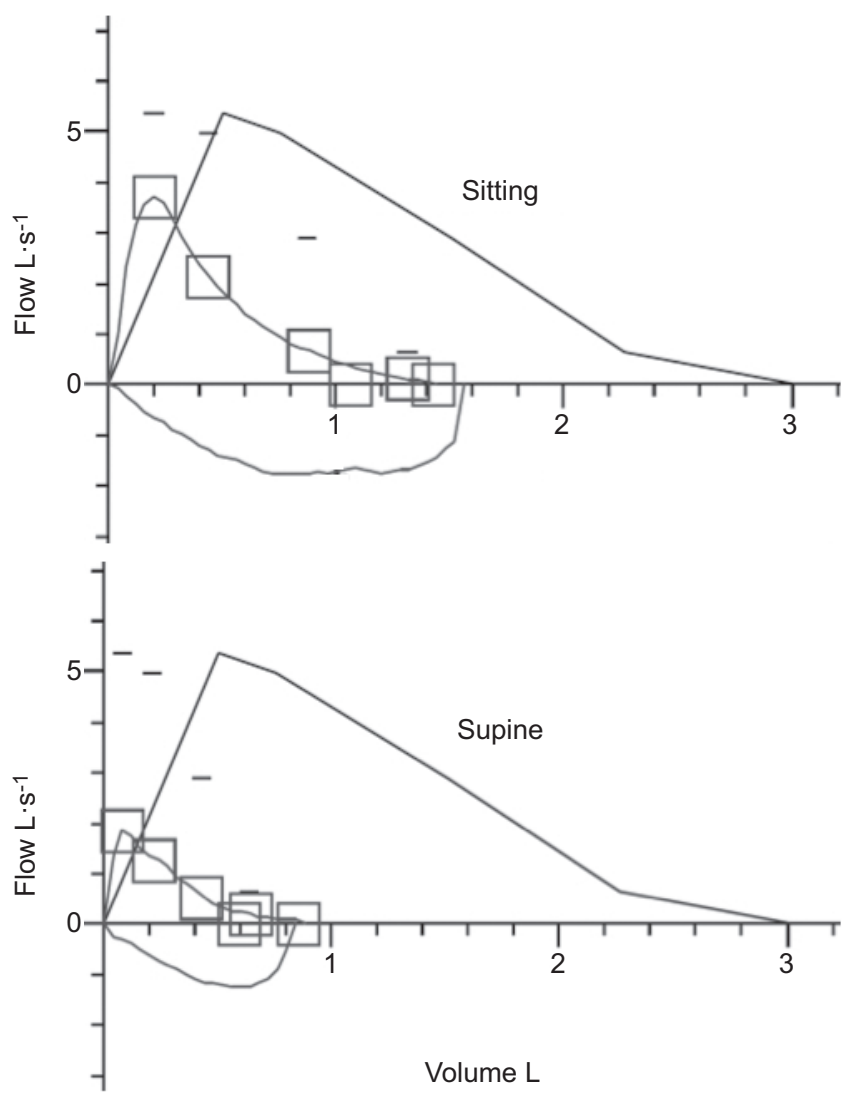

FIGURE 2. Spirometric values in sitting and supine position and the corresponding flow-volume loops. FEV1: forced expiratory volume in $1 \mathrm{~s}$; FVC: forced vital capacity; PI: inspiratory pressure; PE: expiratory pressure; SNIP: sniff nasal inspiratory pressure.

and lung function testing should be performed to rule out highly prevalent ventilatory disorders [8]. However, secondary to in-hospital availability and the trend to parallel rather than linear diagnostic steps, lung function testing was performed after chest CT-scan and was indicated secondary to suspicion of a novel diaphragm paresis in the chest radiograph.

Diagnosis of diaphragm paresis can be made by functional tests such as "sniff" fluoroscopy or spirometry in the sitting and supine position. We performed both tests confirming the diagnosis and supporting our clinical suspicion. In unilateral paresis spirometry usually shows a decrease in vital capacity of $\sim 15-25 \%$ in the supine position compared with the sitting position and in bilateral paresis a decrease of up to $50 \%$ can be observed [9]. An electromyogram using needle electrodes seems not to be mandatory and secondary to the benefit of the NIV and paradoxical breathing pattern in the supine position. The best treatment for dyspnoea secondary to bilateral diaphragm paresis in our case was NIV during bed time. Diaphragm paresis is among the few indications for home ventilation in normocapnic patients [10]. Alternative surgical methods were not applicable because of the advanced local spreading and invasion of the $\mathrm{AE}$.

Our case illustrates that $\mathrm{AE}$ is often diagnosed late because of the slow progression of the disease and often in those cases radical resection is no longer feasible. In endemic areas physicians should be aware of this silent progressive disease because with the increasing fox population and the increasing urbanisation of foxes we will face more clinical cases in the near future.

\section{Philipp Wassmer*, Joannis Chronis*, Jochen J. Rüdiger, and Katia Boggian ${ }^{+}$}

*Dept of Internal Medicine, Cantonal Hospital St Gallen, "Dept of Pneumology, Cantonal Hospital St Gallen, ${ }^{+}$Dept of Infectious Diseases, Cantonal Hospital St Gallen, St Gallen, and "Dept of Biomedicine, University of Basle, Basle, Switzerland.

Correspondence: P. Wassmer, Dept of Internal Medicine, Cantonal Hospital St Gallen, CH-9007 St Gallen, Switzerland. E-mail: philipp.wassmer@kssg.ch

Statement of Interest: None declared.

\section{REFERENCES}

1 Qureshi A. Diaphragm paralysis. Semin Respir Crit Care Med 2009; 30: 315-320.

2 Eckert J, Deplazes P. Biological, epidemiological, and clinical aspects of echinococcosis, a zoonosis of increasing concern. Clin Microbiol Rev 2004; 17: 107-135.

3 Schweiger A, Ammann RW, Candinas D, et al. Human alveolar echinococcosis after fox population increase, Switzerland. Emerg Infect Dis 2007; 13: 878-882.

4 Carmena D, Benito A, Eraso E. The immunodiagnosis of Echinococcus multilocularis infection. Clin Microbiol Infect 2007; 13: 460-475.

5 Brunetti E, Kern P, Vuitton DA, et al. Expert consensus for the diagnosis and treatment of cystic and alveolar echinococcosis in humans. Acta Trop 2010; 114: 1-16.

6 Kadry Z, Renner EC, Bachmann LM, et al. Evaluation of treatment and long-term follow-up in patients with hepatic alveolar echinococcosis. Br J Surg 2005; 92: 1110-1116.

7 Torgerson PR, Schweiger A, Deplazes P, et al. Alveolar echinococcosis: from a deadly disease to a well-controlled infection. Relative survival and economic analysis in Switzerland over the last 35 years. J Hepatol 2008; 49: 72-77.

8 Ray P, Birolleau S, Lefort $\mathrm{Y}$, et al. Acute respiratory failure in the elderly: etiology, emergency diagnosis and prognosis. Crit Care 2006; 10: R82.

9 Gibson GJ. Diaphragmatic paresis: pathophysiology, clinical features, and investigation. Thorax 1989; 44: 960-970.

10 Celli BR. Respiratory management of diaphragm paralysis. Semin Respir Crit Care Med 2002; 23: 275-281.

DOI: $10.1183 / 09031936.00045712$ 